\title{
Análisis cualitativo y cuantitativo para los estudios políticos. Trilateralismo, metodología experimental y Grounded Theory
}

QUALITATIVE AND QUANTITATIVE ANALYSIS FOR POLITICAL STUDIES. TRILATERAL SCIENCE, EXPERIMENTAL METHODOLOGY AND GROUNDED THEORY

Mg. Adolfo Eslava (aeslava@eafit.edu.co) Departamento de Gobierno y Ciencias Políticas, Universidad EAFIT (Medellín, Colombia).

\begin{abstract}
The paper discuss the application of qualitative and quantitative techniques in political studies. It describes the classical proposal of Johan Galtung, known as trilateral science, which invites to the permanent contrast to confirm, validate and accept facts and ideas; then it explores Grounded Theory as a mechanism to achieve and explore data in order to identify a possible dialogue with experimental methodology. Finally, it shows social capital as a case study to demonstrate the relevance of multi-methodological applications.
\end{abstract}

Key words: social capital, Galtung, grounded theory, experimental methodology, trilateral science.

\section{Resumen}

El propósito de este texto es discutir la aplicación de técnicas cualitativas y cuantitativas en los estudios políticos. Para ello, se describe la propuesta clásica de Johan Galtung, conocida como trilateralismo, que invita al contraste permanente con el fin de confirmar, validar y aceptar hechos e ideas; luego, se busca explorar la Grounded Theory como mecanismo de consecución y exploración de datos con el fin de identificar un posible diálogo con la metodología experimental. Por último, se aborda el capital social como objeto de estudio que demuestra la pertinencia de aplicaciones multimetodológicas.

Palabras clave: capital social, Galtung, teoría fundada en datos, metodología experimental, ciencia trilateral. 


\section{Introducción}

Este trabajo hace una reflexión acerca de la necesaria conexión metodológica entre explicación y comprensión, entre dato y relato, asunto para el cual se hace uso de la narración de actores concernidos y se tiende un puente hacia el lenguaje matemático como fuente adicional de argumentación para el análisis político.

El propósito del texto es contribuir a la discusión respecto a la aplicación de técnicas de análisis cualitativo y cuantitativo a problemas propios de los estudios políticos. Para ello, se pretende profundizar en las técnicas de recolección de evidencia empírica sin olvidar que los datos tienen que estar acompañados tanto de explicaciones como de interpretaciones de los mismos.

En primer lugar, se describe la propuesta clásica de Johan Galtung, conocida como trilateralismo, que invita al contraste permanente con el fin de confirmar, validar y aceptar hechos e ideas; luego, se busca explorar la Grounded Theory como mecanismo de consecución y exploración de datos con el fin de identificar un posible diálogo con la metodología experimental que pretende cuantificar fenómenos objeto de estudio. Por último, se aborda el capital social como objeto de estudio que demuestra la pertinencia de la aplicación de técnicas cualitativas y cuantitativas a objetos de estudio de los estudios políticos.

\section{Actividad científica trilateral}

Las ciencias sociales y humanas -en especial, los estudios políticos- están expuestas permanentemente a una realidad cambiante que exige interpretación y cuantificación. En ocasiones el trabajo científico se reduce a la función descriptiva y otras veces se detiene en las cifras exagerando en la formalización, mientras que la sociedad reclama pensadores que den cuenta de la evolución de las complejas interdependencias que la caracterizan y que brinden ideas orientadoras para sus actuaciones colectivas y personales.

El artículo clásico de Galtung (1972) acerca de la relación entre actividad científica y tiempo, plantea la necesidad de concebir el trabajo de la ciencia en el pasado como informador de hechos, en el presente como momento en que el futuro comienza a suceder y en el futuro como escenario por descubrir y crear. En ese contexto temporal, propone la actividad científica trilateral como mecanismo de creación de nuevas realidades.

Según la "actividad científica trilateral", es posible conciliar el mundo observado de los datos, el mundo previsto por la teoría y el mundo preferido por los juicios de valor con base en cinco elementos, a saber:

Análisis Empirismo basado en la concordancia entre dato y teoría

Utopía $\quad$ Formulación de objetivos a partir de los juicios de valor

Realidad Criticismo fundado en la concordancia entre juicio de valor y dato

Propuestas Constructivismo que surge de la concordancia entre juicio de valor y teoría

Acción Concordancia entre dato, teoría y juicio de valor que se traduce en acción creadora de nueva realidad 
Analizar, soñar y chocarse con la realidad para proponer y actuar son actividades propias de la vida cotidiana de las personas. La conciliación de los mundos previstos, preferidos y observados es una tarea posible; tarea en la cual, los datos hacen las veces de punto de partida y de llegada como fuente de retroalimentación del proceso continuo de la actividad científica. Varias décadas después de formulada, la propuesta de Galtung tiene plena vigencia y constituye una exhortación ineludible no sólo para académicos, sino también para tomadores de decisiones en todas las esferas. Es una iniciativa multidisciplinar que invita al contraste permanente con el fin de confirmar, validar y aceptar hechos e ideas. En la triada científica en mención, los datos permiten confirmar la validez de teorías al tiempo que confrontan los juicios de valor. Sin duda, la evidencia es un componente esencial en el trabajo intelectual debido al carácter dinámico que le imprime; nuevas realidades traen consigo revisiones teóricas y matizan algunos juicios de valor. El dato se convierte en factor desencadenante de la acción reformista galtungiana.

La información capturada por un dato tiene que estar enmarcada en un contexto teórico de hipótesis objeto de confirmación y en un contexto valorativo de aserciones objeto de validación. El dato ofrece imágenes de la realidad y se enriquece a partir del diálogo científico con hipótesis y preceptos. Esa triple coincidencia dato-teoría-valor es fuente de propuestas para la acción renovadora de la sociedad como un todo. Los resultados de ingentes esfuerzos dedicados a la investigación académica suelen conformarse con ver la tenue luz de publicaciones de circulación reducida, lo cual las condena a permanecer en los anaqueles del olvido ubicados al margen de los procesos de toma de decisiones. El enfoque integral del trilateralismo brinda las bases para superar el ostracismo científico y situarlo en el lugar que merece como protagonista de la "actividad científica trilateral".

\section{Metodología experimental y trilateralismo}

Existe una controversia interesante respecto al papel de la experimentación en la ciencia política. La visión tradicional ha sido escéptica, pero la creciente utilización de pruebas de laboratorio o grupos de control, encuestas y trabajos de campo unido al apoyo de las innovaciones tecnológicas, han hecho de los experimentos fuente importante de contribuciones en materias sustantivas. La experimentación ha cumplido la mayoría de edad gracias al trabajo de las décadas comprendidas entre mitad de los setenta y mitad de los noventa.

Recientemente se ha definido la ciencia política como una disciplina experimental debido a la amplia variedad de respuestas que la experimentación ha ofrecido para teóricos, empíricos y hacedores de política. En particular, la prominencia experimental se soporta en los avances tecnológicos al servicio de la investigación y en dos razones de fondo: "1) los métodos no experimentales no han podido responder a algunas preguntas de investigación importantes, en particular las causales, y 2) en la actualidad hay nuevas preguntas de investigación de interés para los científicos políticos que son particularmente adecuados para el estudio experimental" (Morton y Williams 2010:10). Alvin Roth (economista galardonado con el Nobel en 2012) identifica tres roles que los experimentos pueden cumplir: "hablar con teóricos" para probar predicciones y supuestos, "susurrar al oído de la princesa" para facilitar el diálogo entre experimentadores y hacedores de políticas respecto al contexto y "buscar hechos" para aislar causas de regularidades observadas y estimar los parámetros causales. 
Es posible establecer una conexión entre la actividad científica trilateral propuesta por Galtung y las audiencias o roles a los que atiende la ciencia experimental. Tanto audiencias como roles apuntan en la dirección de la actividad científica trilateral, en donde el dato, la teoría y el juicio de valor constituyen el trípode donde tiene asiento la actividad científica en general y la ciencia política en particular.

Es posible plantear una nueva relación triangular en la que tiene lugar la ciencia experimental orientada a la búsqueda de mayores niveles de concordancia entre lo previsto, lo preferido y lo observado. Las relaciones entre teóricos, hacedores de política y empíricos tiene lugar bajo el denominador común de la acción creadora de nueva realidad. No se trata de cumplir un rol específico o atender una audiencia en particular, por el contrario la actividad científica, sea experimental o no experimental, debe asumir un enfoque integrador que dé cuenta del consorcio entre dato (buscar hechos), teoría (hablar a teóricos) y juicio de valor ("susurrar a la princesa").

Esta metodología para los estudios políticos no se reduce a los métodos experimentales. También se cuenta con métodos cualitativos para hacer frente al dilema "muchas variables, pocos casos" y con métodos cuantitativos apoyados en instrumental estadístico y econométrico. De acuerdo con los intereses del investigador, el análisis cualitativo puede ser simple o complejo, desde la descripción en lo micro hasta la explicación macropolítica pasando por la investigación cualitativa-comparativa y la causalidad que a su vez encuentra en la GT una herramienta para comprender las múltiples interacciones que se deben considerar. Por su parte, el análisis cuantitativo comprende una amplia gama de opciones, partiendo de la recolección de cifras disponibles hasta el diseño y construcción de indicadores o desde el análisis estadístico univariado hasta innovaciones econométricas.

Se debe promover una suerte de fusión del análisis cuantitativo y cualitativo con la creatividad experimental bajo la etiqueta de caja de herramientas disponible para la acción creadora galtungiana. Los métodos no son de uso excluyente, es decir, la decisión de elegir una de las alternativas disponibles en la caja de herramientas no es obstáculo para acudir a una segunda opción. Al respecto, Ragin concluye que los enfoques comparativos cualitativos tienen que ser completados con otras formas de análisis, en tanto que Druckman afirma que los experimentos son una metodología que puede complementar otros enfoques.

Algunas herramientas disponibles para la metodología política pasa por la descripción, comparación o teoría fundamentada de los ejercicios cualitativos, los indicadores, estadísticas y modelos econométricos del instrumental formal y los mecanismos participativos, juegos económicos, entrevistas y evaluaciones de impacto del método experimental. El presente texto no pretende ser una revisión exhaustiva de los métodos disponibles para el análisis político, por el contrario, solo toma un par de metodologías que en conjunto pueden brindar orientaciones básicas para abordar el estudio de los fenómenos políticos en sintonía con la búsqueda de la convergencia de datos, teorías y juicios de valor. 


\section{Grounded Theory}

En español se conocen varias traducciones de la Grounded Theory [GT]: fundamentada, anclada, aterrizada, enraizada, generada; vocablos que denotan una invitación a mirar hacia el exterior de la teoría. En esencia, el principal mensaje consiste en afirmar que en lugar de plantear hipótesis para verificar en el terreno, es el terreno con su riqueza empírica el que tiene la auténtica posibilidad de hacer preguntas y sugerir hipótesis que, en principio, están fuera del alcance del investigador. De esta forma, el método exige volcarse al objeto de estudio con el fin de obtener grandes volúmenes de información que se somete a diversas técnicas de análisis; en lugar de rastrear categorías analíticas previamente establecidas. El proceso de recopilación de evidencia empírica constituye la principal fuente, no solo de hipótesis, sino también de conceptos pertinentes, relaciones causales y actores concernidos.

Los antecedentes de la GT se pueden resumir en estos tres autores: Glaser y Strauss (1967) fueron los cofundadores de la metodología a partir de las dificultades y hallazgos obtenidos en sus trabajos de investigación sociológica. En el año 1990, el mismo Strauss junto con la doctora en enfermería Juliet Corbin, publicaron la primera versión de un texto dedicado a las técnicas y procedimientos para orientar la aplicación de dicha metodología. A continuación se ofrece una mirada panorámica a este debate de la GT Ilamando la atención respecto a los términos básicos y el procedimiento con el fin de tener una introducción sucinta al caso de estudio.

John Durston sintetiza este método cualitativo con estas palabras: "La metodología de teoría aterrizada rechaza explícitamente la formulación previa de hipótesis y el diseño experimental, como marco de la investigación en terreno. Es inductiva, partiendo de un simple tema de investigación, y un muestreo teórico para buscar excepciones a las regularidades que empiezan a emerger de las entrevistas y observaciones, y la elaboración de relaciones conceptuales complejas entre categorías analíticas en una discusión colaborativa y comparativa de la codificación de cientos de entrevistas" (2001:8).

El propósito de acudir a una metodología como la GT, se sustenta en la riqueza de los hallazgos provenientes de figuras etnográficas como el relato, la entrevista abierta sobre temas simples, la elaboración de mapas y sociogramas; también se plantea la necesidad de contar con la discusión colaborativa entre investigadores con el fin de lograr una codificación participativa acerca de las variables que la realidad sugiere incluir en el estudio. Cabe anotar que la discusión colaborativa está precedida de la elaboración de memos por parte de cada investigador en donde a manera de bitácora, se van consignando apreciaciones, preguntas, conceptos y preguntas que el avance del trabajo de campo inspira en el investigador. En breve, se trata de ganar en la rigurosidad del análisis etnográfico de la mano de lectura deliberada de la evidencia empírica.

Es importante destacar otro aspecto introductorio de la GT que consiste en la adecuada combinación que debe buscar el investigador entre formas cualitativas y cuantitativas. En su trabajo seminal que enuncia la teoría como proceso antes que como producto perfecto, Barney Glaser y Strauss (1) arguyen en favor de la necesidad de ambas formas para la verificación mutua y para la generación de teoría. El punto de partida reside en evitar ubicarse en extremos excluyentes, pero tampoco basta con enunciar su 
complementariedad salomónica. El reto no es otro que lograr una auténtica interacción de métodos. Sin embargo, la GT plantea que tal interacción está precedida del principio de la emergencia de la teoría: antes que comenzar con cifras o conceptos preestablecidos, "se les debe permitir que emerjan durante el proceso de investigación [en este sentido] a veces, puede ser necesario usar medidas cuantitativas; otra veces, la recolección de datos cualitativos y el análisis pueden ser lo más apropiado" (Strauss y Corbin 2002:37).

Tabla 1: Divorcio de los cofundadores

\begin{tabular}{|l|l|l|}
\cline { 2 - 3 } \multicolumn{1}{c|}{} & \multicolumn{1}{c|}{ Glaser } & \multicolumn{1}{c|}{ Strauss y Corbin } \\
\hline $\begin{array}{l}\text { Selección e } \\
\text { identificación del } \\
\text { problema }\end{array}$ & $\begin{array}{l}\text { El problema emerge en el marco del } \\
\text { estudio y depende de las percepciones } \\
\text { conjuntas de actores e investigador }\end{array}$ & $\begin{array}{l}\text { El investigador puede predeterminar } \\
\text { el tema general antes de entrar en } \\
\text { terreno }\end{array}$ \\
\hline $\begin{array}{l}\text { Método de } \\
\text { codificación y análisis }\end{array}$ & $\begin{array}{l}\text { Marco de referencia general basado } \\
\text { en la comparación continua: las } \\
\text { categorías emergen y no pueden ser } \\
\text { forzadas por la metodología }\end{array}$ & $\begin{array}{l}\text { Método analítico con pasos } \\
\text { estructurados: los procedimientos y } \\
\text { técnicas necesitan ser detalladas de } \\
\text { manera precisa }\end{array}$ \\
\hline $\begin{array}{l}\text { Grado de } \\
\text { operacionalización }\end{array}$ & $\begin{array}{l}\text { Bajo debido a las dificultades del } \\
\text { amplio marco de referencia. }\end{array}$ & $\begin{array}{l}\text { Alto dada la especificidad de las } \\
\text { etapas analíticas }\end{array}$ \\
\hline $\begin{array}{l}\text { Naturaleza de la } \\
\text { teoría emergente }\end{array}$ & $\begin{array}{l}\text { Genera conceptos y sus relaciones } \\
\text { para explicar e interpretar las } \\
\text { variaciones de los comportamientos } \\
\text { en el área substantiva estudiada }\end{array}$ & $\begin{array}{l}\text { Genera una teoría derivada de } \\
\text { manera inductiva respecto de un } \\
\text { fenómeno, gracias a conceptos } \\
\text { interrelacionados }\end{array}$ \\
\hline Prueba de la teoría & Análisis cuantitativos subsiguientes & $\begin{array}{l}\text { Prueba provisional para validar } \\
\text { conceptos y sus interrelaciones }\end{array}$ \\
\hline
\end{tabular}

Tomado y adaptado libremente de Raymond (2005)

Por otra parte, Creswell asegura que el desacuerdo entre Glaser y Strauss estriba en la defensa que este último hace de un tipo de GT basada en procedimientos más analíticos y sistemáticos mientras que Glaser evoca la creatividad del; la tercera corriente en contienda se ubica en el enfoque constructivista que propone Kathy Charmaz en su texto de 2006 intitulado Constructing Grounded Theory. Frente a esta alternativa, vale la pena anotar que Glaser critica esta visión constructivista de la [GT], acusándola de privilegiar los intereses de los investigadores y la precisión de sus trabajos, en detrimento de las principales preocupaciones de los investigados. Al revés, la [GT] aparece como una manera de acercarse a la realidad conceptual de los datos, que si existe fuera del papel del investigador (Raymond).

Debido a estas discusiones subyacentes, no es posible hablar de un procedimiento en el que estén de acuerdo los investigadores que trabajan con la teoría fundamentada; no obstante, a continuación se describen las etapas que sintetizan los trabajos de Naresh R. Pandit y Odis E. Simmons. El primero de ellos más apegado a los desarrollos de Strauss en tanto que el segundo declara su visión glaseriana del proceso; una tercera fuente del procedimiento en mención, ubicado en una postura intermedia del debate epistemológico, se encuentra en el texto de autoría de John Creswell. 
De manera sucinta, las fases de los estudios con teoría fundamentada son:

1. Preparación. Mientras la corriente de Glaser intenta minimizar conceptos preestablecidos y pone en duda la conveniencia de revisar la literatura, la postura más sistemática acude a la literatura técnica con el fin de definir la pregunta de investigación y los conceptos preliminares que orientarán el estudio. En esta etapa, existe coincidencia respecto a la necesidad de acudir al muestreo teórico (2) en contraposición al muestreo aleatorio.

2. Recolección de datos. El propósito común es obtener la mayor cantidad de información posible, razón por la cual resultan útiles los instrumentos cualitativos como la entrevista y la observación participante así como las fuentes cuantitativas. La divergencia radica en la necesidad o no de contar con protocolos rigurosos antes del ingreso al trabajo de campo.

3. Ordenación de datos. Ragin afirma que "los investigadores sociales buscan identificar el orden y la regularidad dentro de la complejidad de la vida social" (2007:71), motivo por el cual es indispensable clasificar la información que da cuenta de esa complejidad. Para ello, se propone acudir a diseños cronológicos que permitan distinguir los procesos subyacentes al fenómeno objeto de estudio.

4. Análisis de datos. Aquí se propone el método comparativo constante (3) mediante el uso de las codificaciones abierta, axial y selectiva.

Tabla 2: Codificaciones para analizar los datos

\begin{tabular}{|l|l|}
\hline \multicolumn{1}{|c|}{ Codificación } & \multicolumn{1}{c|}{ Definición } \\
\hline Abierta & $\begin{array}{l}\text { Proceso analítico por medio del cual se identifican los conceptos y se descubren en } \\
\text { los datos sus propiedades y dimensiones (pág. 110). }\end{array}$ \\
\hline Axial & $\begin{array}{l}\text { Proceso de relacionar las categorías a sus subcategorías, denominado axial porque } \\
\text { la codificación ocurre alrededor del eje de una categoría, y enlaza las categorías en } \\
\text { cuanto a sus propiedades y dimensiones (pág. 134). }\end{array}$ \\
\hline Selectiva & Proceso de integrar y refinar la teoría (pág. 157). \\
\hline
\end{tabular}

Elaboración propia con base en Strauss y Corbin (2002).

5. Desarrollo de teoría. Dado que el propósito central es desarrollar y no probar teorías, el investigador comienza sin teoría alguna o bien, con una teoría que desea modificar, con el fin de fundamentar su estudio en los puntos de vista de los participantes en el trabajo de campo.

6. Saturación. Las categorías están soportadas en tantos incidentes, eventos y actividades como sea posible recoger; el proceso llega a su saturación en el momento en que no es posible encontrar nueva información que mejore la comprensión de la categoría.

El proceso descrito hace énfasis en la necesidad de concebir el ejercicio como una tarea cíclica que puede comenzar y recomenzar. Sin embargo, también es preciso aclarar que no se trata de someter los estudios a la inconveniente lógica lineal de etapas precedidas unas de otras; por el contrario, el propósito es resaltar 
la condición de un proceso que está sujeto a la retroalimentación permanente de acuerdo con los hallazgos que cada etapa puede ir arrojando, pero sobre todo, por las relaciones causales inferidas, el análisis comparativo, la deliberación colectiva y las contribuciones de los investigados. En resumen, el procedimiento constituye un método serio de análisis que le da cabida a la aparición de la serendipity (4).

En términos del trilateralismo descrito en la primera sección, el procedimiento de la GT interviene en el lado de los datos, de los juicios de valor y de la teoría. La conexión entre teoría fundamentada y actividad científica trilateral se evidencia cuando la GT se define como "una teoría derivada de datos recopilados de manera sistemática y analizados por medio de un proceso de investigación. En este método la recolección de datos, el análisis y la teoría que surgirá de ellos guardan estrecha relación entre sí" (Strauss y Corbin 2002:13).

En suma, la GT es actividad científica trilateral puesto que a pesar de su énfasis claramente cualitativo, los instrumentos de investigación también permiten construir teorías con base en juicios de valor de los actores concernidos y en los órdenes de magnitud acerca de las condiciones en las que tiene lugar el fenómeno social objeto de estudio. Las etapas de recopilación, ordenación y análisis de datos traen consigo la posibilidad de acumular un acervo de información que combina cuantificación de hechos con cualificación de preferencias con el fin de construir la teoría fundamentada en evidencia empírica.

\section{Aplicación al estudio del capital social}

Con base en las movilizaciones mundiales del 2011, Joan Subirats hace una propuesta provocadora que se resume en la invitación a trascender las voces de indignación respecto a las diferentes formas de representación política, con el fin de construir una democracia de lo común. En este sentido, el intelectual catalán asegura que por debajo y en la periferia de la política actual se ha ido tejiendo una urdimbre cívica fundamentada en las lógicas y en los bienes relacionales que es necesario reconocer desde mecanismos distintos a las respuestas técnicas; al respecto, destaca "la necesidad de incorporar opiniones legas en temas de complejidad técnica relevante pero que son difícilmente resolubles sin esa visión de no-experto" (Subirats 2011:64).

Esa exhortación tiene hondas repercusiones conceptuales y empíricas. La complejidad de esa urdimbre cívica para comprender lo común constituye un desafío metodológico de enormes dimensiones. A continuación se describe una aproximación a esa dimensión civil desde el capital social comunitario, asunto en el cual confluye la actividad científica trilateral aquí expuesta. En primer lugar, se aborda la teoría fundamentada en datos como estrategia metodológica para abordar el fenómeno objeto de estudio y a continuación se plantean algunas ideas de metodología experimental que pueden complementar el ejercicio inicial.

Recordemos que el capital social es un concepto que puede ofrecer respuestas a cuestiones sociales, como la señalada por Subirats, así como a los dilemas colectivos que ha estudiado Elinor Ostrom; sin embargo, aún tiene muchos obstáculos por resolver tanto desde lo teórico y metodológico como desde su observación empírica. 
Existen varias contribuciones a la comprensión del término por parte de autores reconocidos como James Coleman, Pierre Bourdieu y Robert Putnam. En Bernardo Kliksberg es posible encontrar un panorama completo de autores e ideas que resume el concepto en función de cuatro dimensiones constitutivas, a saber: confianza, espíritu cívico, asociatividad y valores. John Durston también hace una revisión completa del término, para luego proponer el capital social comunitario entendido como "la institucionalidad formal e informal que integra las normas culturales de confianza entre individuos, por un lado, con las prácticas de cooperación entre todos los miembros de un sistema social" (2000:24).

De esta forma, la cuestión por el capital social trae consigo la discusión acerca de la pertinencia de los métodos cuantitativos y cualitativos y de su carácter excluyente o complementario, por ejemplo, la siguiente cita ilustra el debate: "La cuantificación siempre exige un sacrificio de información compleja para la ganancia de precisión; en el caso del capital social, todavía hay una necesidad prioritaria de investigaciones empíricas cualitativas que arrojen nuevas hipótesis de trabajo más finas, que puedan emerger de hallazgos no anticipados y que den cuenta de toda la complejidad de los fenómenos interrelacionados que caben bajo el rótulo de capital social" (Durston 2001:5).

Como se anotó arriba, no existe un procedimiento único para llevar a cabo un estudio inscrito en la GT. Por ejemplo, Durston hace uso de esta metodología pero con los ajustes que exige su objeto de estudio: Teoría pre-existente (Discusión colaborativa - Distanciar del terreno) $\rightarrow$ Datos (Entrevistas - Relatos en detalle) $\rightarrow$ Comparación iterativa (Generar hipótesis - De vuelta al terreno), todo lo cual se puede describir con las palabras del propio autor cuando asegura que la GT mantiene los marcos teóricos pre-existentes lo más lejos posible de la investigación en terreno: "usa entrevistas abiertas sobre un tema simple de estudio, en vez de delimitar variables independientes y dependientes y excluir el resto de la información del entorno. Seguimos este principio básico en el primer terreno. Pero no renunciamos a nuestro interés en la discusión teórica sobre capital social y nuestras conversaciones comparativas siempre estuvieron alimentadas por esta literatura. De hecho, la iteración entre comparación, generación de hipótesis y vuelta al terreno implicó la formulación de cuestionamientos a la literatura. El creciente desafío de distanciarse de los marcos académicos fue contestado principalmente por la técnica de grabar relatos lo más detallada y exhaustivamente posible, limitando el papel de la reflexión teórica a la discusión colaborativa, la elaboración de una lista de temas a explorar y al muestreo teórico" (Durston 2001:8).

La aplicación al caso de la evaluación de capital social comunitario, además de insistir en la complementariedad entre cifras y conceptos, también hace manifiesta una apuesta por los matices. Si bien se declara que el estudio es una aplicación de la GT, la exposición del método, sus instrumentos y hallazgos, muestran la necesidad de no apegarse estrictamente ni a conceptos ni a procedimientos previamente establecidos. En particular, es importante destacar el papel que juega un equipo interdisciplinario de trabajo en la investigación. A mi modo de ver, el énfasis en la discusión colaborativa enriquece el estudio y permite obtener hallazgos a partir de la interpretación colectiva de la evidencia empírica, lo cual, constituye una contribución que Durston le hace a la metodología. Este tipo de discusión intensiva y comparativa hace las veces de filtro valioso que usan los investigadores para poner en común sus memorandos -reflexiones y pre-hipótesis de trabajo- y someterlos a la revisión crítica de otras lecturas 
de la evidencia recopilada. Todo en ello, con el propósito de retornar al trabajo de campo con nuevas preguntas y nuevas claves interpretativas, de tal manera que al final sea posible concluir que: "Es posible dar rigor científico a este procedimiento y elaborar modelos cada vez más sofisticados -aunque se trate de cuestiones teóricas nuevas- mediante la recopilación frecuente de datos cualitativos y la formulación de nuevas hipótesis para guiar nuevas preguntas en terreno" (Durston 2002:128).

El proceso de las diferentes codificaciones conduce a la formulación de un modelo teórico que dista de las categorías iniciales. Como se observa en la Tabla 3, en el caso del capital social, se pasa de cuatro a seis modalidades que permiten una mejor comprensión de las vivencias comunitarias y que Durston (2002) sintetiza en estos términos:

Individual. Las relaciones tienen el carácter de un contrato informal, con contenido de confianza y reciprocidad. Esta forma de capital social no reside en la persona misma sino en las relaciones.

Grupal. Se trata de personas que tienen mucha confianza unas en otras, porque han acumulado múltiples experiencias de reciprocidad difusa.

Comunitario. El capital social comunitario reside no sólo en el conjunto de las redes de relaciones interpersonales diádicas, sino en las estructuras que forman la institucionalidad de cooperación comunitaria, es decir, en el sistema sociocultural propio de cada comunidad, en sus estructuras de gestión y sanción.

Puente. Vínculos extensos horizontales que se establecen entre actores de similar poder. Permite a las comunidades y organizaciones -sectoriales, territoriales o poblacionales- tender puentes entre sí, lo cual amplía la comunidad y el grado de confianza, dado que su principal fuerza está en la unión.

Escalera. Reciprocidad con control asimétrico. Conecta a un actor de escaso poder verticalmente con otro de mayor poder y puede servir para empoderar y desarrollar sinergias gracias al acceso a recursos económicos, políticos o cognitivos.

Societal. Hace hincapié en las virtudes o debilidades de las culturas nacionales dotadas de sistemas normativos integradores. Ayuda a analizar el modo en que las políticas públicas pueden apoyar a algunas comunidades en la acumulación de capital social, como factor de su empoderamiento y de la coproducción de mejoras en su calidad de vida. Para esto, es imprescindible ahondar en lo relativo a la interacción entre las instituciones socioculturales del Estado y estos capitales intangibles, sean individuales o colectivos, de las comunidades focalizadas. 
Tabla 3: Codificaciones Durston

\begin{tabular}{|c|c|}
\hline Categorización inicial & Codificación selectiva \\
\hline $\begin{array}{l}\text { INDIVIDUAL } \\
\text { ("contratos diádicos", redes) }\end{array}$ & $\begin{array}{l}\text { CAPITAL SOCIAL INDIVIDUAL } \\
\text { contratos diádicos y redes egocentradas, apoyos } \\
\text { externos, contactos y clientelismos }\end{array}$ \\
\hline $\begin{array}{l}\text { GRUPAL } \\
\text { ("círculos de confianza", facciones) }\end{array}$ & CAPITAL SOCIAL GRUPAL \\
\hline $\begin{array}{l}\text { COMUNITARIO Y ORGANIZACIONAL } \\
\text { (sistema de autogestión, control social) }\end{array}$ & CAPITAL SOCIAL COMUNITARIO Y DE BARRIO \\
\hline \multirow{3}{*}{$\begin{array}{l}\text { EXTERNO } \\
\text { (puente, escalera) }\end{array}$} & $\begin{array}{l}\text { CAPITAL SOCIAL DE PUENTE } \\
\text { eslabonamiento regional y nacional }\end{array}$ \\
\hline & CAPITAL SOCIAL DE ESCALERA \\
\hline & $\begin{array}{l}\text { CAPITAL SOCIAL SOCIETAL } \\
\text { normas e instituciones generalizadas }\end{array}$ \\
\hline
\end{tabular}

Elaboración propia con base en Durston (2001).

En efecto, la GT permite traducir intuiciones e intereses investigativos en un modelo teórico más completo y más cercano a la realidad, debido a que la fuente de elaboración de tal modelo fue el trabajo de campo que logró combinar la indagación experta con la opinión no experta basada en la vivencia.

Hasta aquí se aprecia la ventaja de acudir a un método cualitativo como la GT. No obstante, en consonancia con la actividad científica trilateral es necesario complementar estos hallazgos con ejercicios cuantitativos, por ejemplo como los métodos experimentales aplicados recientemente en Colombia, veamos.

En muchas ocasiones se acude al capital social como concepto que articula las intervenciones sociales pero su medición constituye un verdadero reto en términos de la conversación metodológica señalada arriba entre el explicar y el comprender. A continuación se describe una propuesta para hacerle frente a tal desafío. Entendiendo capital social como aquel capital enfocado en lo que la gente hace más que en lo que posee y que hace alusión a la confianza, la generosidad y la cooperación (Bowles y Gintis).Resulta preciso indagar por las normas de prosocialidad planteadas por estos autores y que, con base en la metodología propuesta e implementada por el Departamento Nacional de Planeación de Colombia (DNP), pueden incluir los atributos señalados en la tercera columna de la Tabla 4.

Tabla 4: Ejercicios de confianza y bien público

\begin{tabular}{|l|l|l|}
\hline \multicolumn{1}{|c|}{ Situaciones } & \multicolumn{1}{|c|}{ Atributos } & \multicolumn{1}{c|}{ Indicador } \\
\hline \multirow{2}{*}{$\begin{array}{l}\text { Nivel } \\
\text { interpersonal }\end{array}$} & Confianza & \% de dinero enviado del jugador 1 al 2 \\
\cline { 2 - 3 } & Reciprocidad & \% de dinero enviado del jugador 2 al 1 \\
\cline { 2 - 3 } & Filantropía & \% donado a una organización local \\
\hline \multirow{2}{*}{ Dilema social } & Cooperación & \% de contribución al bien público \\
\cline { 2 - 3 } & Comunicación & Aumento en contribuyentes cuando existe comunicación \\
\hline
\end{tabular}


Para ello, DNP realiza los ejercicios experimentales descritos arriba, pero con algunas modificaciones que reflejan una aproximación al capital social "entendido como manifestación de modos de relación "virtuosos» en la comunidad" (Departamento Nacional de Planeación 2008:33); tales ejercicios se describen como sigue:

El primero, se aproxima al concepto de confianza, reciprocidad y filantropía al nivel interpersonal. En el segundo se simula una decisión colectiva de inversión en los bienes públicos, y se busca medir la propensión a cooperar en dilemas sociales cuando los intereses individuales y colectivos son diferentes; la dinámica realizada permite también explorar el poder de la comunicación para la construcción de acciones.

Otra alternativa metodológica consiste en implementar el juego que Juan Camilo Cárdenas denomina solución descentralizada comunitaria, que precisamente indaga por el papel de las preferencias prosociales o preferencias por los demás incorporando "la posibilidad de que los individuos transformen los pagos materiales del juego en valoraciones subjetivas donde no solo el bienestar material individual es tenido en cuenta, sino también aspectos asociados al altruismo y al nivel de justicia o igualdad del resultado" (2009:53). Respecto a este tipo de preferencias -que se traducen en reciprocidad, aversión a la inequidad, envidia y altruismo-, Bowles afirma que: "Al elegir cómo actuar, los individuos comúnmente toman en cuenta no sólo las consecuencias de sus acciones para ellos mismos, sino también para otros. Más allá de eso, en general no sólo se preocupan por las consecuencias sino también por las intenciones de los otros actores" (2010:III.5)

\section{Tabla 5: Algunos resultados}

\begin{tabular}{|c|c|}
\hline \multirow{4}{*}{$\begin{array}{l}\text { Juego de } \\
\text { la } \\
\text { confianza }\end{array}$} & $\begin{array}{l}\text { De acuerdo con Karl Sigmund, Ernst Fehr y Martin Nowak, dos de cada tres ofertas de los } \\
\text { jugadores } a \text { oscilan entre el } 40 \% \text { y el } 50 \% \text { del monto a distribuir, en tanto que más de la } \\
\text { mitad de los jugadores } b \text { rechazan la oferta cuando ésta es inferior al } 20 \% \text {. }\end{array}$ \\
\hline & $\begin{array}{l}\text { El experimento de Cárdenas, Chong y Ñopo (aplican en seis ciudades capitales de } \\
\text { América Latina) concluye que "la oferta promedio fue } 43 \% \text { de la dotación inicial y la } \\
\text { mediana fue } 50 \% \text { (en el } 32 \% \text { de las decisiones)" (2009:61). }\end{array}$ \\
\hline & $\begin{array}{l}\text { El estudio de DNP (2008), aplicado en } 42 \text { municipios colombianos, muestra que el } \\
\text { indicador de confianza es } 58 \% \text { y el de reciprocidad igual a } 64 \% \text {. Además la filantropía } \\
\text { asciende a } 40 \% \text {. }\end{array}$ \\
\hline & $\begin{array}{l}\text { El estudio realizado en el departamento de Antioquia, Colombia, asegura que "la } \\
\text { confianza de los antioqueños, medida por el juego experimental es de un } 51 \% \text {. Esto se } \\
\text { traduce en que en el juego de confianza, el promedio de dinero enviado del jugador } 1 \text { al } \\
\text { jugador } 2 \text { equivale al } 51 \% \text { de la dotación inicial" (Giraldo, Casas, Méndez y Eslava } \\
2013: 81 \text { ). }\end{array}$ \\
\hline \multirow{3}{*}{$\begin{array}{l}\text { Juego del } \\
\text { bien } \\
\text { público }\end{array}$} & $\begin{array}{l}\text { De acuerdo con Cárdenas, uno de cada cuatro participantes decidió contribuir al bien } \\
\text { público. }\end{array}$ \\
\hline & $\begin{array}{l}\text { Con base en DNP (2008), el nivel de cooperación es igual a } 63 \% \text { y la existencia de canales } \\
\text { de comunicación incrementa la cooperación en cerca de siete puntos porcentuales. }\end{array}$ \\
\hline & $\begin{array}{l}\text { El estudio realizado en el departamento de Antioquia, Colombia, asegura que "El nivel de } \\
\text { disposición a la acción colectiva de los antioqueños es de un } 40 \% \text {. Esto quiere decir que }\end{array}$ \\
\hline
\end{tabular}


el $40 \%$ de los participantes decidió poner su ficha en la cuenta de grupo y el $60 \%$ restante optó por los beneficios privados de quedarse con ella" (Giraldo, Casas, Méndez y Eslava 2013:82).

Los resultados recogidos en la Tabla 5 coinciden en rechazar la hipótesis de cero confianza o cero cooperación, que trae consigo el supuesto del comportamiento humano que solo toma decisiones en términos de obtener la mayor utilidad posible para sí mismo; por el contrario, los experimentos ofrecen un orden de magnitud respecto a la convivencia de actores racionales con otra variedad de actores, toda vez que como he argumentado a propósito de los móviles de la conducta humana: "otras motivaciones están presentes en la mente y el corazón humanos; aunque el interés personal nos mueve a comportarnos de una u otra manera, por fortuna en el devenir humano tiene lugar el interés por otros, fundado en la simpatía o el compromiso, como factor crucial de nuestras decisiones" (Eslava 2010:251) .

Los hallazgos permiten identificar cómo se han resuelto o qué disponibilidad existe para contribuir a la solución de problemas de acción colectiva propios de la construcción y uso de capital social. De esta forma, es posible contar con mejor información no sólo para diseñar los incentivos adecuados, sino también para incorporar la cooperación arraigada en el comportamiento auténtico de las personas, de tal forma que sea posible lograr una mejor forma de las políticas.

En breve, el reto consiste en crear y encontrar las condiciones bajo las cuales se pueden tomar decisiones con base en el acervo proveniente de los atributos de la comunidad. En la medida que el investigador aborde mejor las condiciones en cuestión, es posible también estudiar los factores de éxito o fracaso a lo largo del proceso de las políticas. Identificar y estimar los niveles de prosocialidad puede ofrecer respuestas acerca de cuestiones claves de la creación, acumulación y uso del capital social.

\section{Conclusión}

Se dice que la investigación cualitativa hace énfasis en las personas y en su vida social, e indaga por discursos y significados, de tal manera que ha cobrado relevancia el denominado giro argumentativo en la investigación científica. Se trata de un campo de trabajo abierto y dinámico que enriquece la labor académica en contraposición a la ausencia de apertura que se le endilga a la mirada positivista.

Es común encontrar discusiones metodológicas que se sustentan en ver la discusión cualitativacuantitativa como antinomia. La una se enorgullece de sus fortalezas en términos comparativos frente a las características de la otra y no es exagerado afirmar que la una se define en contraposición a la otra.

Nada más ajeno al imperativo del diálogo de saberes. Un metodólogo cuantitativo, a pesar de su obsesión por los datos, también interpreta; de igual manera el metodólogo cualitativo también establece relaciones cuantificables entre sus narraciones. En breve, a pesar de la categorización en la que incurre el académico, no olvidemos que la realidad exige tender puentes entre esas categorías. Al respecto, una voz autorizada en el método experimental asegura: "El reto para quienes tratamos de pensar los problemas y las políticas públicas está en la construcción de esos puentes. Podemos enriquecer nuestra estrategia de investigación buscando y construyendo estos puentes entre los niveles macro y micro, y entre la modelación teórica y 
la investigación empírica. El reto incluye mucho más que la interdisciplinariedad, y mucho más que la construcción de la transdisciplinariedad. Se tratar de dialogar entre formas de enfrentar el mundo y sus problemas desde cada disciplina y el papel de la academia, el estado, la sociedad civil, los gremios, o los grupos en conflicto" (Cárdenas 2002:42).

Finalmente, cabe anotar que al momento de hablar de los métodos, no es posible plantear afirmaciones en blanco o negro. No es conveniente buscar rasgos inequívocos pues el diseño de la investigación, aún bajo todo el peso del rigor científico, no permite aseveraciones acerca de la mayor pertinencia de un método en función de problemas planteados, teorías existentes, actores concernidos e hipótesis. Por el contrario, la pregunta de investigación tiene que estar formulada de tal manera que permita aprovechar la riqueza emanada de la multiplicidad de métodos disponibles.

\section{Nota}

(1) A manera de antecedente, cabe señalar que mientras Strauss provenía de la tradición de investigación sociológica cualitativa de Chicago, Glaser obtuvo su posgrado en la Universidad de Columbia en donde la investigación hacía uso de innovaciones en los métodos cuantitativos.

(2) Es una muestra de individuos objeto de estudio que comienza con un conjunto homogéneo y, a medida que avanza la investigación y emergen categorías, el investigador lo convierte en una muestra heterogénea con el fin de observar bajo qué condiciones se mantiene la validez de las categorías (Creswell). Charles Ragin aclara que no se trata de un asunto de conveniencia puesto que "[e]l fin del muestreo teórico no es realizar un muestreo con el fin de capturar todas la variaciones posibles, sino más bien para ayudar al desarrollo de los conceptos y profundizar en la comprensión de los objetos de investigación" (2007:171).

(3) Técnica de análisis cualitativo para ayudar a la formulación y depuración de conceptos que implica un diálogo continuo entre ideas y pruebas empíricas, de esta forma, las comparaciones establecen similitudes y diferencias que ayudan a definir o modificar los conceptos y las categorías (Ragin).

(4) Con base en las ideas de Robert K. Merton, Charles Tilly define este concepto como "un mecanismo en el que interactúan ambiente y conocimiento para producir un descubrimiento significativo e inesperado" (2010: 55).

\section{Bibliografía}

Bowles, S. 2010. Microeconomía. Comportamiento, instituciones y evolución. Bogotá: Uniandes.

Cárdenas, J.C. 2002. Sistemas naturales y sistemas sociales: hacia la construcción de lo público, lo colectivo, lo ambiental. Bogotá: Colciencias.

http://wwwprof.uniandes.edu.co/ jccarden/DialogEstratResumen.pdf 
Cárdenas, J.C. 2009. Dilemas de lo colectivo. Instituciones, pobreza y cooperación en el manejo local de los recursos de uso común. Bogotá: Uniandes.

Cárdenas, J.C., Chong, A. y Ñopo, H. 2009. To what extent do Latin Americans trust, reciprocate, and cooperate? Evidence from experiments in six Latin American countries. Economía 9(2): 45-88. DOI: 10.1353/eco.0.0027

Departamento Nacional de Planeación. 2008. Evaluación de impacto de los programas Paz y Desarrollo y Laboratorios de Paz. Bogotá: DNP-Sinergia.

Durston, J. 2000. ¿Qué es el capital social comunitario? Santiago: CEPAL. http://www.cepal.org/publicaciones/xml/5/4885/lcl1400.pdf

Durston, J. 2001. Evaluando capital social en comunidades campesinas en Chile. Santiago: Asesorías para el Desarrollo. asesoriasparaeldesarrollo.cl/docs/473079415.pdf

Durston, J. 2002. El capital social campesino en la gestión del desarrollo rural. Díadas, equipos, puentes y escaleras. Santiago: CEPAL. http://archivo.cepal.org/pdfs/2002/S2002033.pdf

Eslava, A. 2010. La idea de la justicia al servicio del desarrollo en Amartya Sen. Co-herencia 7(13): 245-260. http://www.redalyc.org/articulo.oa?id=77416997010

Galtung, J. 1972. Empiricism, criticism, constructivism. Three approaches to scientific activity. Synthese 24 (3/4): 343-372. DOI: 10.1007/BF00413652

Giraldo, J; Casas, A; Eslava, A. y Méndez, N. 2013. Valores, representaciones y capital social en Antioquia. Medellín: EAFIT - SURA - Gobernación de Antioquia.

Glaser, B.G. y Strauss, A. 1967. The discovery of grounded theory: strategies for qualitative research.Chicago: Aldine.

Morton, R.B. y Williams, K.C. 2010. Experimental political science and the study of causality: from nature to the lab. Cambridge: Cambridge University Press.

Ragin, C. 2007. La construcción de la investigación social. Introducción a los métodos y su diversidad. Bogotá: Siglo del Hombre - Uniandes.

Raymond, E. 2005. La teorización anclada (grounded theory) como método de investigación en ciencias sociales: en la encrucijada de dos paradigmas. Cinta moebio 23: 217-227.

Strauss, A. y Corbin, J. 2002. Bases de la investigación cualitativa. Técnicas y procedimientos para desarrollar la teoría fundamentada. Medellín: Universidad de Antioquia.

Subirats, J. 2011. Otra sociedad, ¿otra política? De "no nos representan" a la democracia de lo común.Barcelona: Icaria.

http://www.icariaeditorial.com/pdf libros/otra\%20sociedad\%20otra\%20politica.pdf 
Tilly, C. 2010. Mechanisms of the middle range. En: C.J. Calhoun (ed.) Robert K. Merton: sociology of science and sociology as science. New York: Columbia University Press, pp. 54-62. http://essays.ssrc.org/tilly/wp-content/uploads/2008/06/tilly-mechanisms-middle-range.pdf

Recibido el 8 Mar 2014

Aceptado el 11 Sep 2014 